\title{
The combined effects of etanercept plus methylprednisolone on pancreatic oxidative stress in acute pancreatitis induced by cerulein
}

\author{
Murat Günay*a, Erol Kılıç ${ }^{\mathrm{b}}$, Ramazan Amanvermez \\ ${ }^{a}$ Department of Biochemistry, Medical Faculty, Ondokuz Mayis University, Samsun, Turkey \\ ${ }^{b}$ Department of General Surgery, Medical Faculty, Ondokuz Mayis University, Samsun, Turkey
}

\begin{tabular}{|c|c|}
\hline \multicolumn{2}{|c|}{ ARTICLE INFO } \\
\hline \multicolumn{2}{|c|}{ Article History } \\
\hline Received & $04 / 03 / 2011$ \\
\hline Accepted & 14 / 03 / 2011 \\
\hline
\end{tabular}

\section{* Correspondence to:}

Murat Günay

Ondokuz Mayıs University,

Medical Faculty, Department of

Biochemistry, Samsun, Turkey

e-mail:drmuratgunay@yahoo.com

\begin{tabular}{l}
\hline Keywords \\
Etanercept \\
Methylprednisolone \\
Acute pancreatitis \\
Oxidative stress \\
Malondialdehyde \\
Protein carbonyls
\end{tabular}

\begin{abstract}
Whether etanercept plus metylprednisolone reduce pancreatic oxidative stress and injury during pancreatitis is unknown well. The aim of this study was to examine the therapeutic effects of etanercept and methylprednisolone (MP) on acute pancreatitis and oxidative stress in acute pancreatitis (AP) induced by cerulein in a rat model. The study was performed with 48 rats divided into 6 groups ( $n=8 /$ group): 1-sham, 2-cerulein-induced pancreatitis (over 10 hours), 3- cerulein-pancreatitis (over 20 hours), 4-etanercept (5 mg/ $\mathrm{kg}$, i.p.), 5-methylprednisolone (10 mg/kg, i.m.), 6-etanercept plus methylprednisolone. Also, the rats in groups 4, 5, and 6 were cerulein-induced pancreatitis at 20 hours. After the treatment, the pancreas and blood were taken for histopathological and biochemical analysis. All cerulein-treated rats developed biochemical and pathological acute pancreatitis after 10-20 hours. The markers of oxidative stress such as protein carbonyls, lipid peroxidation, and myeloperoxidase (mpo) in the pancreas tissues were increased in the group 2 and 3, but these were lower in etanercept and MP-treated rats compared to groups 3. Pancreatic mpo activity was considerably reduced in pancreas tissues at $20 \mathrm{~h}$ after the administration of etanercept plus metylprednisolone in the group 6 compared to group 3. In AP induced by cerulein, the treatment of etanercept plus methylprednisolone may ameliorate pancreatic oxidative stress in rats.

J. Exp. Clin. Med., 2011; 28:12-16
\end{abstract}

(c) 2011 OMU All rights reserved

\section{Introduction}

The etiological basis of AP is multifactorial (Steer, 2008). Usually AP known as a pancreatic disease is an inflammation initially localized in the pancreatic tissue which may cause to local and systemic complications in the patients. The mortality due to AP is approximately $10 \%$, but this estimate raise up to $20-30 \%$ in the severe forms of the disease due to multiple organ failure. Progress in medicine, mostly supportive and antibiotic therapy and surgery all has contributed to reduce mortality and morbidity rates in severe AP in the past decades (Pereda et al., 2006; Bang et al., 2008; Steer, 2008). However, no specific effective treatment has been reported in the clinical trials so far.

There is considerable evidence that the inflammation contributes significantly to the developing pancreatic damage by mechanisms like infiltration of inflammatory cells (granulocytes, monocytes/macrophages) and the release of toxic substances such as reactive oxygen species produced in excess, lipid peroxidation, protein oxidation and their harmful compounds, and pro-inflammatory cytokines (e.g. IL-1, IL-6 and TNF- $\alpha$ ) (Reinheckel et al., 1998; Kyu et al., 2003; Pereda et al., 2006). Actived macrophages release pro-inflammatory cytokines in response to the local damage of the pancreas. Moreover, increased oxidative stress appeared during pancreatitis in the course of AP (Dabrowski et al., 1999; Telek et al., 1999; Abu-Zidan et al., 2000; Shi et al., 2005). At present there are evidences of a synergy or the relationship between oxidative stress and pro-inflammatory cytokines in development of the inflammatory response in AP (Pereda et al., 2004; Pereda et al., 2006). In relation to this issue, Pereda et al have shown that simultaneous inhibition of TNF- $\alpha$ production and xanthine oxidase activity blocks to a great extent the local and systemic inflammatory response in AP (Pereda et al., 2004). In addition, inflammation and oxidative stress both occur in the pancreas during the early stages of acute pancreatitis (Dabrowski et al., 1999).

It seems that inflammation is a dominant phenomenon in the early stage of AP and during the course of the disease. The patient's acute pancreatitis may be resolved whether pancreatic inflammation could be blocked or reduced by anti-inflammatory agents. Effective therapeutic agents are required to reduce morbidity and mortality rate of acute pancreatitis associated life loss. Etanercept which is a soluble TNF- $\alpha$ binding agent, competitively inhibits the binding of TNF 
molecules to TNF receptor sites. The binding of etanercept to TNF renders the bound TNF biologically inactive, resulting in a significant reduction in inflammatory activity (Malleo et al., 2007). Like most corticosteroids, methylprednisolone is typically used for its anti-inflammatory effects. The function of etanercept plus MP is unclear as a therapeutic agent in the prevention and treatment of AP (Bang et al., 2008). The purpose of present study was to investigate whether the combined effects of etanercept plus MP could prevent, or decrease pancreatic oxidative stress in cerulein-induced AP, as defined by the markers of oxidative stress.

\section{Materials and Methods}

Sprague-Dawley male rats, weighing 250-270 g were used in all experiments. The rats were housed in per cage with wood chip bedding and fed on standard laboratory chow and water ad libitum. They were maintained on a $12 \mathrm{~h}$ light: dark cycle with a constant room temperature at $22 \pm 1{ }^{\circ} \mathrm{C}$. This study was carried out in the Experimental Research Center, Ondokuz Mayis University, Samsun, Turkey. The local ethical committee of Ondokuz Mayis University approved all animal procedures, and the experimental protocol.

Animal treatment: The rats were randomized into six groups $(\mathrm{n}=8 /$ group $)$ : 1 . Sham-treated in which identical treatments to group 2 were performed, except that the saline was administered instead of cerulein; 2. Cerulein-induced pancreatitis (at $10 \mathrm{~h}$ ): Rats were treated hourly (x5) with cerulein (total $80 \mu \mathrm{g} / \mathrm{kg}$, i.p., suspended in saline solution) and an additional 5 hours was allowed to pass for a complete pacreatitis induction at $10 \mathrm{~h} ; 3$. Cerulein-pancreatitis (at $20 \mathrm{~h}$ ): Identical to the group 2, an extra 10 hours was waited for the continuation of induced pacreatitis; 4. Etanercept group, except for the administration of etanercept $(5 \mathrm{mg} / \mathrm{kg}$, i.p., dissolved in saline solution) which was given hourly (two times) at the end of 5 th $\mathrm{h}$ after the last cerulein application; 5. Methylprednisolone group, MP $(10 \mathrm{mg} / \mathrm{kg}$, i.m. $)$ was given hourly (two times) at the end of 5 th $\mathrm{h}$ after the last cerulein application. 6. Etanercept plus methylprednisolone group, the administration of methylprednisolone $(10 \mathrm{mg} / \mathrm{kg}$, i.m.) along with etanercept ( $5 \mathrm{mg} / \mathrm{kg}$, i.p.) which were given hourly (two times) at the end of 5 th $\mathrm{h}$ after the last cerulein application. At the end of experiments, the rats were anaesthetised by intramuscular injection of ketamine. After blood samples were obtained by direct intra-cardiac puncture with an injector, all rats were sacrificed by exsanguinations under anaesthesia at $10 \mathrm{~h}$ (group 2) and $20 \mathrm{~h}$ (groups 3,4,5, and 6) after the induction of pancreatitis. Also rats were killed at $20 \mathrm{~h}$ in the group 1. The pancreas was resected with midline laparotomy under sterile conditions. A segment of pancreas was washed in a cooled isotonic sodium chloride solution, dried with filter paper and the pancreatic samples were stored until examination at $-70{ }^{\circ} \mathrm{C}$, and other part of the pancreas was fixed in $10 \%$ buffered formaldehyde for histopathological examination.

Induction of pancreatitis by cerulein: Experimental acute pancreatitis was induced by cerulein injection $(16 \mu \mathrm{g} /$ $\mathrm{kg}$, i.p.) five times at $1 \mathrm{~h}$ intervals, and used in the first part of this research (Takaoka et al., 2002). Injection of cerulein in rats was associated with significant increases in the serum levels of pancreatic amylase and lipase and resulted in acute mild to moderate pancreatitis at 10 and $20 \mathrm{~h}$. Histopathological evaluation of pancreas sections at these hours after ceru- lein administration revealed tissue damage characterized by oedema, vacuolisation, inflammation and cell necrosis. No elevation in the serum values of pancreatic amylase and lipase, and histopathological alterations was observed in pancreatic tissue from sham-treated group compared to groups 2 and 3.

Histopathological study: A part of pancreas was fixed with 24 hours buffered neutral formalin and then processed for the routine tissue follow-up. The tissues were embedded in paraffin blocks and cut into 4-6 $\mu \mathrm{m}$ thick sections, and then stained with Hematoxylin \& Eosin. The stained sections were evaluated by a pathologist who was uninformed about the groups. The method of Rongione et al., (1997) was used for scoring the degree of oedema, inflammation, vacuolization, necrosis in the pancreas.

Biochemical measurements: The markers of oxidative stress marked in this study include protein carbonyls, a marker of protein oxidation; malondialdehyde, a marker of lipid peroxidation; and myeloperoxidase, a neutrophil enzyme that produces oxidants. To determine tissue concentrations of lipid peroxidation, protein carbonyls, myeloperoxidase and protein, a part of rat pancreas was excised, weighed and it was minced and homogenized well in liquid nitrogen immediately after excision, and then the homogenate was sonicated for 60 $\mathrm{s}$ at $220 \mathrm{~V}$ (Fisher, Sonic Dismembrator; Model 300) in each cold assay buffer. All chemicals used for the assays were of analytical grade and purchased from Sigma Chemical Co.

Table 1. The levels of biomarkers of oxidative stress in pancreas tissue in the groups

\begin{tabular}{|l|l|l|}
\hline Groups & $\begin{array}{l}\text { Protein Carbonyls } \\
(\mathrm{nmol} / \mathrm{mg} \text { protein })\end{array}$ & $\begin{array}{l}\text { MDA } \\
(\mu \mathrm{mol} / \mathrm{mg} \text { protein })\end{array}$ \\
\hline 1. $(\mathrm{n}=8)$ & $0.385 \pm 0.163$ & $0.097 \pm 0.032$ \\
\hline 2. $(\mathrm{n}=8)$ & $0.605 \pm 0.218$ & $0.320 \pm 0.208$ \\
\hline 3. $(\mathrm{n}=8)$ & $0.804 \pm 0.299$ & $0.353 \pm 0.183$ \\
\hline 4. $(\mathrm{n}=8)$ & $0.733 \pm 0.138$ & $0.208 \pm 0.076$ \\
\hline 5. $(\mathrm{n}=7)$ & $0.578 \pm 0.171$ & $0.138 \pm 0.023$ \\
\hline 6. $(n=8)$ & $0.513 \pm 0.103$ & $0.112 \pm 0.016$ \\
\hline
\end{tabular}

In the pancreatic homogenate, lipid peroxidation was estimated spectrophotometrically by the tiobarbituric acid-reactive substance (TBARS) method with slight modifications (Singh et al., 2000) and expressed in terms of malondialdehyde (MDA) formed per mg protein. Measurements of protein (carbonyl) oxidation in the pancreas homogenate samples were made by the method of Evans et al., (1999) with slight modifications. Carbonyl concentration (nmol mg-1 of protein $=$ nmol ml-1 of carbonyl groups/protein concentration in mg ml-1. The usefulness of measuring mpo activity to assess neutrophil infiltration has been previously reported (Genovese et al., 2006), the pancreatic homogenate levels of mpo were determined by using hydrogen peroxide as a substrate for the enzyme. One unit of mpo activity is defined as that degrading $1 \mu \mathrm{mol}$ of peroxide per minute at $25^{\circ} \mathrm{C}$ and is expressed in units per $\mathrm{mg}$ protein. The protein concentration was determined by the method of Lowry et al., (1951).

Statistical analysis: Data were controlled for normality assumption, they were normally distributed therefore parametric statistical analysis were used to evaluate data. So, the 
statistical evaluations were done by Univariate Analysis of Variance. Variances were homogeneously distributed according to Levene test, and then the pairwise comparisons were done by Turkey or Tamhane test. Results were expressed as mean \pm standard deviation. Differences were considered statistically significant at $\mathrm{P}$ values $<0.05$.

\section{Results}

Effects of etanercept plus MP on pancreatic oxidative stress during experimental AP.

Levels of pancreatic protein oxidation and lipid peroxidation were found to be lower in pancreas tissue from the animals in the group 6 with the application of etanercept plus MP than those of the group 3 (Table 1). An evidence of AP is inflammation that is related to the accumulation of inflammatory cells in the pancreas, which augment the tissue damage. Therefore, we have determined higher inflammation and necrosis in the group 3 compared to shame-treated group. The activity of mpo is a quantitative measure of neutrophil infiltration in pancreas tissue. The levels of mpo was significantly higher in acute pancreatitis group than that of the sham-treated group $(\mathrm{P}<0.001)$. The activity of mpo was considerably suppressed by etanercept plus MP treatment (Fig 1. ).

After acute pancreatitis induction, protein oxidation and lipid peroxidation were increased during acute pancreatitis. Therefore, oxidative stress markers in pancreas and serum concentrations of amylase and lipase were much higher and pancreatic lesions more severe in acute pancreatitis induced by cerulein in group 3 . However, their levels were attenuated by the administration of etanercept plus MP in group 6 .

\section{Discussion}

Acute pancreatitis is an inflammatory process in the pancreas, and oxygen free radicals have been implicated as an important factor in the associated tissue destruction in both the initiation and progression of AP (Telek et al., 1999; Kyu et al., 2003; Shi et al., 2005; Genovese et al., 2006). In addition, oxidative stress was documented in pancreatic tissue by means of methods showing accumulation of products of lipid peroxidation and occurrence of oxidatively modified proteins, with concomitant depletion of enzymatic and low molecular weigh antioxidants (Reinheckel et al., 1998; Dabrowski et al., 1999). Abu-Zidan et al. and Kiziler et al. have indicated that oxidative stress is a feature within the first $24 \mathrm{~h}$ of the onset of AP (Abu-Zidan et al., 2000; Kiziler et al., 2008).

In scientific literature, there are many experimental evidences of a synergy between oxidative stress and proinflammatory cytokines in the development of the inflammatory response in AP (Denham et al., 1997; Pereda et al., 2004; Pereda et al., 2006). Moreover, pro-inflammatory cytokines and oxidative stress trigger common signal transduction pathways that lead to amplification of the inflammatory cascade (Shi et al., 2005; Pereda et al., 2006). Furthermore, pro-inflammatory cytokines, in particularly TNF- $\alpha$, and oxidative stress promote each other generating a vicious circle in AP. Because TNF- $\alpha$ is a cytokine produced in response to many stimuli and also induces acute inflammation by enhancing endothelial permeability, inflammatory cell recruitment and release of oxygen free radicals and additional cytokines by polymorphonuclear leukocytes (Granell et al., 2004). In previous reports, different anti-TNF- $\alpha$ strategies have been evaluated in experimental pacreatitis. Inhibition of TNF- $\alpha$ attenuated the disease severity and improved survival in experimental models of AP (Hughes et al., 1996; Denham et al., 1997). Etanercept, a novel anti-TNF- $\alpha$ agent, which binds specifically to TNF and blocks its interaction with cell surface TNF receptors, has reduced neutrophil infiltration into pancreas and the development of AP in mice (Malleo et al., 2007).

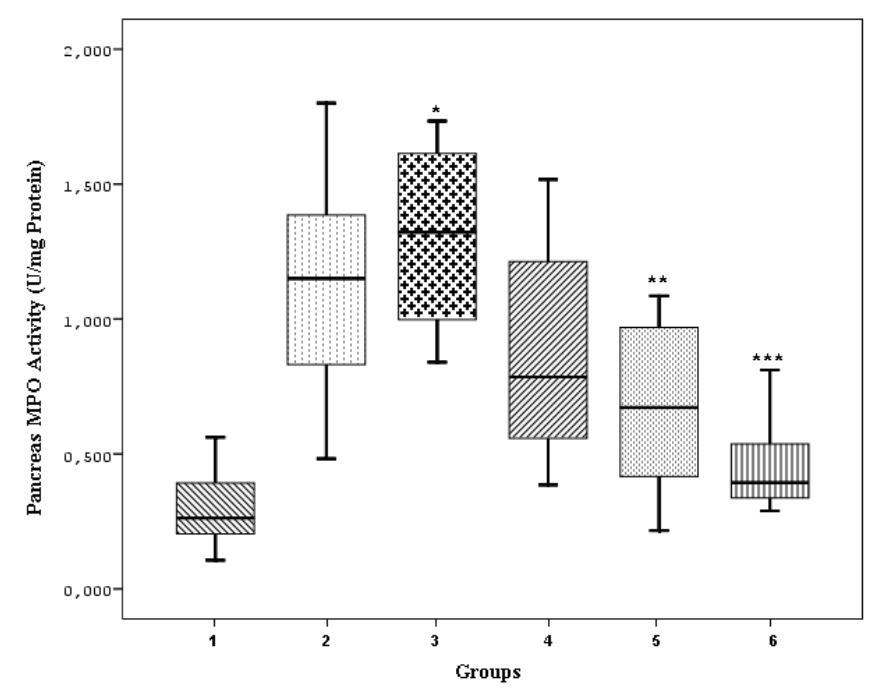

Fig.1. Effects of etanercept and MP administration on pancreatic myeloperoxidase activities in the course of AP. ${ }^{*} \mathrm{P}<0.001$ vs. sham-treated rats. ${ }^{*} \mathrm{P}<0.05$ and $* * * \mathrm{P}<0.001$ vs. ceruleinpacreatitis rats (group 3).

Reactive oxygen species have extensive effects on tissue damage, resulting lipid and protein oxidation and denaturation of enzymes and proteins. These causes damage of the cell membrane and proteolytic digestion of cell components, resulting in cell death and increased vascular permeability with tissue edema (Dean et al.,1997; Reinheckel et al., 1998; Kyu et al., 2003). It has been shown that the levels of oxidative damage markers MDA and protein carbonyls are increased in tissue and plasma in acute pancreatitis (Kiziler et al., 2008). In our study, protein oxidation and lipid peroxidation were found to be higher in the groups 2 and 3 compared to shamtreated group. By the way, pancreatic protein carbonyls and MDA levels were lower by $8.83 \%$ and $41 \%$ in etanercepttreated rats than those of the group 3 . Also, the activity of mpo seems to be lower in the groups 4, 5 and 6 received treatments than those of acute pancreatitis groups. In an experimental study, Yilmaz et al. (2009) reported that etanercept caused marked decreases in pancreatic tissue MDA levels and mpo activities in treated groups with taurocholate-induced acute pancreatitis. In according to these findings, TNF- $\alpha$ reduction by etanercept might lead to ameliorate pancreatic oxidative stress in AP.

Corticosteroids are widely used for the suppression of inflammatory responses (Barnes, 1998). Currently these drugs are being used in a wide variety of inflammatory diseases affecting many organs. Kimura et al. reported that glucocorticoids were attenuated pancreatic damage by protecting acinar cells during cerulein-induced AP (Kimura et al., 1998). MP (5-40 mg/kg, 6-72 h) pre-treatment reduced the serum amylase activity, the pancreatic levels of TNF- $\alpha$ and IL- 6 , the pancreatic mpo activity and morphological parameters of the 
disease during CCK-induced AP in rats (Rakonczay et al., 2003). In our study, MP at the dose of $10 \mathrm{mg} / \mathrm{kg}$ in ceruleininduced AP was led to be decreased pancreatic protein oxidation (by 28.1\%) and lipid peroxidation (by 60.9\%) levels in the group 5 compared to group 3 (Table 1). Similarly, the activity of mpo was reduced significantly in the group 5 given MP. As a result of these findings, MP can have a protective effect against pancreatic oxidative stress and damage in AP. Takaoka et al., (2002) indicated that the beneficial effects of MP arose both from the inhibition of the local inflammation in the pancreas and the blockade of generalized inflammation through the activation of a cytokine cascade and hemodynamic disturbance as well.

In the present study, we hypothesized whether treatment with etanercept plus MP could be given in the early stage of pancreatitis; the long-term effects or the pancreatic oxidative stress during of the disease might be decreased or prevented. As cited in literature: If direct correlation between oxidative stress and severity of pancreatitis have been reported4, etanercept and MP exert potent anti-inflammatory effects in AP (Rakonczay et al., 2003; Malleo et al., 2007). In our study, pancreatic protein carbonyls and MDA values were decreased in the pancreas at $20 \mathrm{~h}$ after the treatment of these drugs. Apart from these results, the activity of mpo was statistically reduced in the group 6 compared to group 3 (Table 1 and Fig. 1). Based on these results we hypothesize that etanercept plus MP influences the oxygen radical formation and minimizes protein oxidation and lipid peroxidation. Inflammatory cells in particularly neutrophils which play a crucial role in the development and full manifestation of AP, are very active in this disease (Genovese et al., 2006). Oxidative stress may be closely associated with inflammatory process and the severity of AP (Kyu et al., 2003). The antioxidative effects of these drugs may partly be due to inhibition of neutrophil chemotaxis. Possibly, etanercept plus MP therapy in inflamed pancreatic tissue may result in a significant reduction in inflammatory activity. Our previous study report has indicated that a decrease in pancreas damage histopathologically when using etanercept and MP for pancreatic injury in ceruleininduced AP (Kilic et al., 2010).

In conclusion, these results support that the treatment of etanercept plus MP cause the attenuation of pancreatic oxidative stress in cerulein-induced AP. Based on the results of recent studies, it is mentioned that treatments of etanercept and MP have preventive effects on AP development and its complications. So they may be useful in biliopancreatic surgery and endoscopic retrograde cholangiopancreatography (ERCP). The combination of etanercept plus MP might be useful in the therapy of conditions associated with AP as the drugs of protective against pancreatic damage. Studies from different areas involving high number of animals, pro-inflammatory cytokines like TNF- $\alpha$ and the other oxidative stress markers are needed to confirm the findings of the present study.

\section{Acknowledgements}

The authors thank Dr. Leman Tomak for providing the statistical advice.

\section{REFERENCES}

Abu-Zidan, F.M., Bonham, M.J.D., Windsor, J.A. 2000. Severity of acute pancreatitis: a multivariate analysis of oxidative stress markers and modified Glasgow criteria. Br. J. Surg. 87, 1019-1023.

Bang, U.C., Semb, S., Nojgaard, C., Bendtsen, F. 2008. Pharmaclogical approach to acute pancreatitis. World J. Gastroenterol. 14, 29682976.

Barnes, P.J. 1998. Anti-inflammatory actions of glucocorticoids: molecular mechanisms. Clin. Sci. 94, 557-572.

Dabrowski, A., Konturek, S.J., Konturek, J.W., Gabryelewicz, A. 1999. Role of oxidative stress in the pathogenesis of cerulean-induced acute pancreatitis. Europ. J. Pharmacol. 377, 1-11.

Dean, R.T., Fu, S., Stocker, R., Davies, M.J. 1997. Biochemistry and pathology of radical-mediated protein oxidation. Biochem. J. $324,1-18$. Denham, W., Fink, G., Yang, J., Ulrich, P., Tracey, K., Norman, J. 1997. Small molecule inhibition of tumor necrosis factor gene processing during acute pancreatitis prevents cytokine cascade progression and attenuates pancreatitis severity. Am. Surg. 63, 1045-1049.

Evans, P., Lyras, L., Halliwel, B. 1999. Measurement of protein carbonyls in human brain tissue. Methods Enzymol. 300, 145-156.

Genovese, T., Mazzon, E, Di Paola, R., Muia, C., Crisafulli, C., Menegazzi, M., 2006. Hypericum perforatum attenuates the development of cerulein-induced acute pancreatitis in mice. Shock. 25, 161-167.

Granell, S., Pereda, J., Gomez-Cambronero, L., Cassinello, N., Sabater, L., Closa, D., 2004. Circulating TNF- $\alpha$ and its soluble receptors dur ing experimental acute pancreatitis. Cytokine. 25,187-191.

Hughes, C.B., Gaber, L.W., Mohey el-Din, A.B., Grewal, H.P., Kotb, M., Mann, L.A.O.1996. Inhibition of TNF- $\alpha$ improves survival in an ex perimental model of acute pancreatitis. Am. Surg. 62, 8-13.

Kilic, E., Amanvermez, R., Kefeli, M., Polat, C., Gunay, M. 2010. Protective effects of etanercept and methylprednisolone on pancreatic dam agein cerulein-induced acute pancreatitis. Saudi Med. J. 31, 394-399.

Kimura, K., Shimosegawa, T., Sasano, H., Abe, R., Satoh, A., Masamune, A., 1998. Endogenous glucocorticoids decrease the acinar cell sensi tivity to apoptosis during cerulein pancreatitis in rats. Gastroenterology. 114, 372-381.

Kiziler, A.R., Aydemir, B., Gulyasar, T., Unal, E., Günes, P. 2008. Relationships among iron, protein oxidation and lipid peroxidation levels in rats with alcohol-induced acute pancreatitis. Biol. Trace Elem Res.124, 135-143.

Kyu Park, B., Chung, J.B., Lee, J.H., Hun Suh, J., Woo Park, S., Song, S.Y., 2003. Role of oxygen free radicals in patients with acute pancreati tis. World J. Gastroenterol. 9, 2266-2269.

Lowry, O.H., Rosebrough, N.J., Farr, A.L., Randal, R.J. 1951. Protein measurement with the folin phenol reagent. J. Biol. Chem. 193, 265275.

Malleo, G., Mazzon, E., Genovese, T., Paola, R.D., Muia, C., Centorrino, T., 2007. Etenarcept attenuates the development of cerulein-induced acute pancreatitis in mice: A comparison with TNF- $\alpha$ genetic deletion. Shock. 27, 542-551.

Pereda, J., Sabater, L., Aparisi, L., Escobar, J., Sandoval, J., Vina, J.2006. Interaction between cytokines and oxidative stress in acute pancreatitis. Current Med. Chemistry 13, 2775-2787.

Pereda, J., Sabater, L., Cassinello, N., Gomez-Cambronero, L., Closa, D., Folch-Puy, E., 2004. Effect of simultaneous inhibition of TNF- $\alpha$ production and xanthine oxidase in experimental acute pancreatitis. Ann Surg. 240, 108-116.

Rakonczay, Z., Duda, E., Kaszaki, J., Ivanyi, B., Boros, I., Lanovics, J., 2003. The anti-inflammatory effect of methylprednisolone occurs down- 
stream of nuclear factor-kB DNA binding in acute pancreatitis. Eur. J. Pharmacol. 464, 217-227.

Reinhecke,l T., Nedelev, B., Prause, J., Augustin, W., Schulz, H.U., Lippert, H., 1998. Occurrence of oxidatively modified proteins: an early event in experimental acute pancreatitis. Free Rad. Biol. Med. 24, 393-400.

Rongione, A.J., Kusske, A.M., Kwan, K., Ashley, S.W., Reber, H.A., McFadden, D.W. 1997. Interleukin-10 reduces the severity of acute pan creatitis in rats. Gastroenterology. 112, 960-967.

Shi, C., Andersson, R., Zhao, X., Wang, X. 2005. Potential role of reactive oxygen species in pancreatitis-associated multiple organ dysfunc tion. Pancreatology. 5, 492-500.

Singh, R.P., Padmavathi, B., Rao, R. 2000. Modulatory influence of Adhatoda vesica leaf extract on the enzmes of xenobiotic metabolism, anti oxidant status and lipid peroxidation in mice. Mol. Cell Biochem. 213, 99-109.

Steer, M.L.2008. Exocrine pancreas. In: Tavsend CM, Beauchamp RD, Evers BM, Mattox KL (18th ed./eds). Sabiston textbook of surgery: the biological basis of modern surgical practice. Philadelphia: Saunders, Elsevier inc.1589 - 1623.

Takaoka, K., Kataoka, K., Sakagami, J. 2002. The effect of steroid pulse therapy in the development of acute pancreatitis induced by closed duodenal loop in rats. J. Gastroenterol. 37, 537-542.

Telek, G., Scoazec, J-Y, Chariot, J., Ducroc, R., Feldmann, G., Roze, C. 1999.Cerium-based histochemical demonstration of oxidative stress in taurocholate-induced acute pancreatitis in rats: a confocal laser scanning microscopic study. J. Histochem. Cytochem. 47, 1201-1212.

Y1lmaz, M., Topsakal, S., Herek, O., Ozmen, O., Sahinduran, S., Buyukoglu, T., 2009. Effects of etanercept on sodium taurocholate-induced acute pancreatitis in rats. Translational Res. 154, 241-249. 